\title{
Electronic Structures and Induced-Hole Carriers of Covalent Semiconductors in External Electric Field*
}

\author{
Aiichiro Sugiyama, ${ }^{\dagger}$ Kohji Nakamura, Kazuhiro Sano, Toru Akiyama, and Tomonori Ito \\ Department of Physics Engineering, Mie University, \\ 1577 Kurima-machiya, Tsu, Mie 514-8507, Japan
}

(Received 13 December 2013; Accepted 5 February 2014; Published 15 March 2014)

\begin{abstract}
To investigate electric-field-induced superconductivity in covalent semiconductors, band structures and inducedhole carrier density of hydrogenated $\mathrm{Si}(110)$ surface in an external electric field were calculated by using first principles full-potential linearized augmented plane-wave method. Results predict that the induced-hole carriers, which form a Fermi surface with a hole pocket centered at $\Gamma$ point, are less than the critical density of superconductivity for boron-doped Si. Thus, electric-field-induced superconductivity at $\mathrm{Si}(110)$ surface may be difficult to achieve, which is in contrast to the case of diamond(110) surface. [DOI: 10.1380/ejssnt.2014.109]
\end{abstract}

Keywords: Electric field; Hole carriers; Silicon; Density functional calculations; Superconductivity

\section{INTRODUCTION}

Controlling carrier density in covalent semiconductors is one of the key issues in the fields of semiconductor physics and engineering. In diamond, carrier doping up to $10^{21} \mathrm{~cm}^{-3}$ was succeeded by boron incorporation. It turns the system to be metallic and to excite superconductivity with superconducting transition temperature $\left(T_{\mathrm{c}}\right)$ of $11.4 \mathrm{~K}[1,2]$. Superconductivity in the other covalent semiconductors, e.g., boron-doped $\mathrm{Si}$ and $\mathrm{SiC}$ with $T_{\mathrm{c}}$ of $0.35 \mathrm{~K}[3]$ and $1.4 \mathrm{~K}[4]$, respectively, was further reported experimentally. In order to increase $T_{\mathrm{c}}$, more efforts to introduce high carrier doping will be practically demanded. In the chemical doping, however, structural disorders always cause an unnecessary complexity in physical properties and may suppress the $T_{\mathrm{c}}[5-7]$.

Alternately, electric-field-driven superconductivity at insulator surfaces recently has the subject of considerable attention [8], where an application of an external electric field that induces high carrier density opens an advanced way for searching and investigating superconductivity in insulating materials without the chemical doping. Previously [9], we investigated the electric-field-driven superconductivity in hydrogenated diamond(110) surface from first principles calculations, and found that an introduction of a negative electric field that induces hole carriers results in a metallic surface with accumulated hole carriers exceeding the critical carrier density responsible for superconductivity of boron-doped diamond [1].

Here, we extend first principles investigations for treating covalent semiconductors including hydrogenated $\mathrm{Si}(110)$ surface by means of first principles full-potential linearized augmented plane-wave (FLAPW) method [1012]. Results predict that when an external electric field is introduced, the system turns to be metallic, where Fermi level $\left(E_{\mathrm{F}}\right)$ locates the top of valence band around the $\Gamma$ point and the Fermi surface with a hole pocket is formed. In contrast to the diamond case, however, the induced

\footnotetext{
*This paper was presented at the 12 th International Conference on Atomically Controlled Surfaces, Interfaces and Nanostructures (ACSIN-12) in conjunction with the 21st International Colloquium on Scanning Probe Microscopy (ICSPM21), Tsukuba International Congress Center, Tsukuba, Japan, November 4-8, 2013.

$\dagger$ Corresponding author: $412 \mathrm{~m} 606 @ \mathrm{~m} \cdot \mathrm{mie}-\mathrm{u} . \mathrm{ac} \cdot \mathrm{jp}$
}

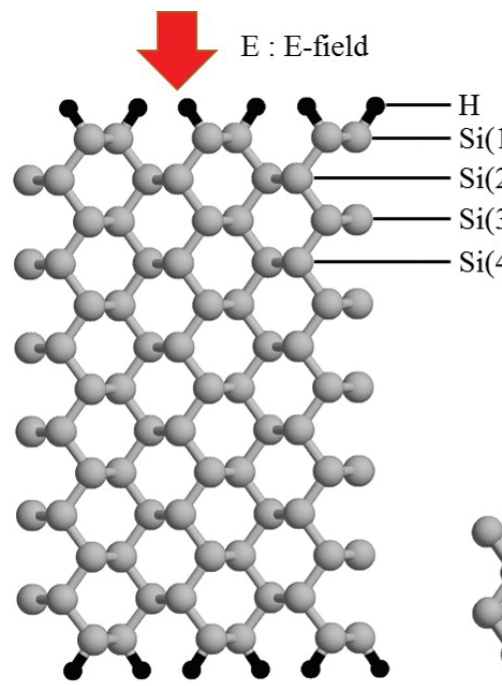

(a)Side view

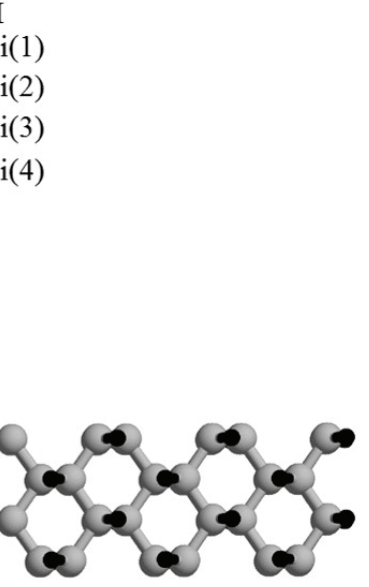

(b)Top view
FIG. 1: Atomic structure of hydrogenated Si(110) surface, (a) side view and (b) top view. The single slab consists of $\mathrm{Si}$ 13 layers (gray circles) and is terminated by hydrogens (black small circles) on both sides of the slab.

hole carriers are found to be less than the critical density for superconductivity of boron-doped Si.

\section{METHOD AND MODEL}

We consider the hydrogenated $\mathrm{Si}(110)$ surface, modeled as a single slab consisted of 13 atomic-layers, which is terminated by hydrogens on both sides of the slab, as shown in Fig. 1. In-plane lattice constants are assumed to match to those of bulk $\mathrm{Si}$, and all atomic positions are fully optimized by the atomic force calculations in an electric field.

Calculations were carried out by using FLAPW method based on the local density approximation [13]. LAPW basis with a cut-off of $|k+G| \leq 3.9$ a.u. ${ }^{-1}$ and muffintin (MT) sphere radii of 1.40 a.u. for Si and 0.65 a.u. for $\mathrm{H}$ atoms are used. Lattice harmonics with angular momenta up to $l=8$ for $\mathrm{Si}$ and 6 for $\mathrm{H}$ are employed to expand the charge density, potential, and wave functions. 


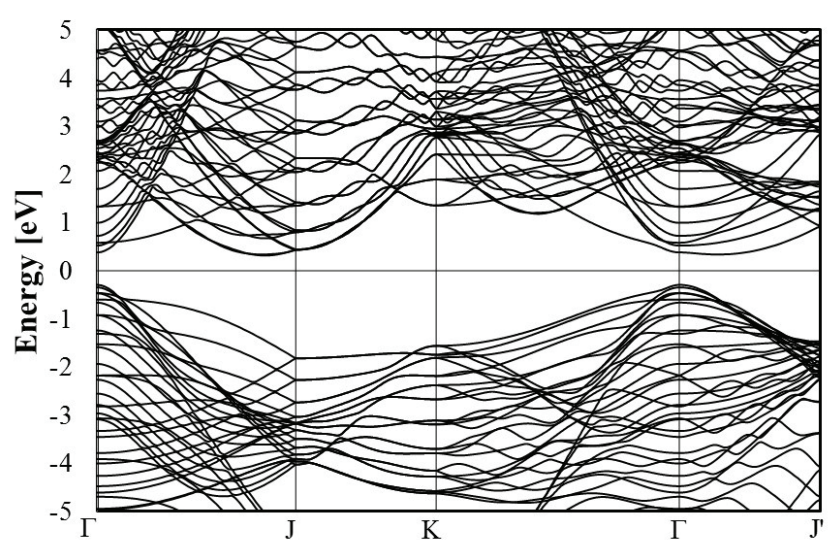

FIG. 2: Calculated band structure for Si (110) surface in zero external electric field. The energy gap is estimated to $0.61 \mathrm{eV}$.

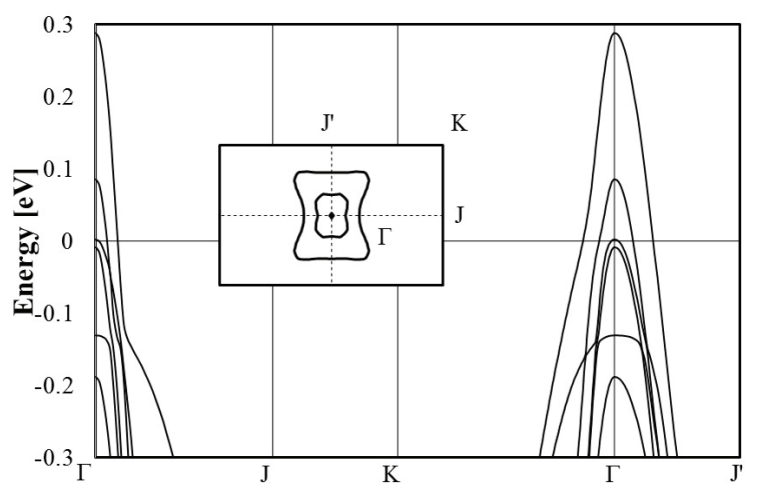

FIG. 3: Calculated band structure for Si (110) surface in an external electric field of $-1.0 \mathrm{~V} / \AA$. Fermi energy, $E_{\mathrm{F}}$, locates in the valence bands at $\Gamma$ point. The reference energy $(E=$ $0)$ places $E_{\mathrm{F}}$. In inset, Fermi surface in the external electric field is presented, where the Fermi surface with a hole pocket centered at $\Gamma$ point appears.

$24 \times 16$ special $k$-points in two-dimensional Brillouin zone (BZ) for self-consistent calculations were employed. To introduce an external electric field along perpendicular to the surface, we place an electrode (a sheet of charge) far enough outside surface so that electrons have negligible overlap with the sheet $[14,15]$.

\section{RESULTS AND DISCUSSION}

We first present atomic and electronic structures of hydrogenated $\mathrm{Si}(110)$ surface in zero field. The hydrogen terminations on the $\mathrm{Si}(110)$ surface, with the H-Si(1) bond length of $1.52 \AA$, remove dangling bonds, which recovers almost bulk like $s p^{3}$ bonding geometry at the $\mathrm{Si}(110)$ surface. Also, as seen in the band structure in Fig. 2, the hydrogen terminations remove surface states arising from the dangling bonds that appear in the band gap in the clean surface. The system shows a semiconducting feature with an indirect band gap of $0.61 \mathrm{eV}$.

When an external electric field of $-1.0 \mathrm{~V} / \AA$ is introduced, the bond lengths are slightly compressed. The

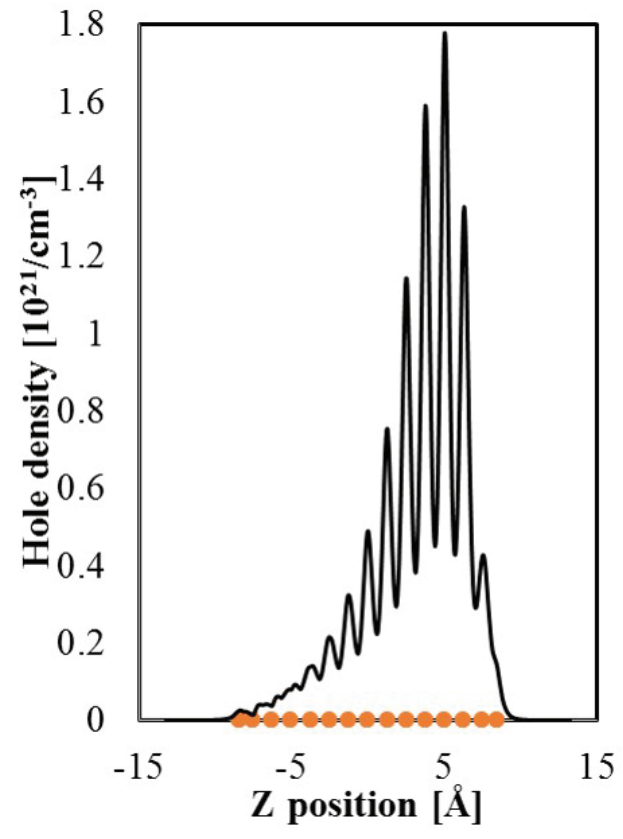

FIG. 4: Calculated hole density as a function of the $z$-position (along normal to surface plane) for Si (110) surface in an external electric field of $-1.0 \mathrm{~V} / \AA$. Solid circles on the horizontal axis represent positions of Si layers.

surface Si-H bond length decreases by $0.007 \AA$ from that in zero field. For the layers below the surface, very small shortening in the bond lengths was observed, where the maximum change appears between the second and third Si layers ( $\mathrm{Si}(2)-\mathrm{Si}(3)$ in Fig. 1) by $0.008 \AA$. In contrast, as shown in Fig. 3, the surface becomes metallic where the $E_{\mathrm{F}}$ locates at the top of valence bands at the $\Gamma$ point, and the Fermi surface with a hole pocket centered at the $\Gamma$ point appears. We confirmed that the area of the Fermi surface increases when the external electric field increases.

Calculated hole carrier density as a function of the $z$ position (along the surface normal) is shown in Fig. 4. This is demonstrated by the planar-averaged hole density along the $z$ axis, corresponding to the states in energy range from the $E_{\mathrm{F}}$ to the top of valence band. Although the hole carriers are mainly induced in a few atomic-layers below the surface, they are rather dispersed inside the film, compared to that in diamond [7]. The highest value of the hole density results in $1.1 \times 10^{21} \mathrm{~cm}^{-3}$ at the $\operatorname{Si}(2)$ layer, which is less than that in boron-doped $\mathrm{Si}$ in experiments, $4.0 \times 10^{21} \mathrm{~cm}^{-3}[3]$.

The mechanism of superconductivity for boron-doped Si may be likely to be electron-phonon coupling $[3,16]$. According to ab initio calculations with both virtual crystal and direct supercell calculations, the superconductivity of boron-doped $\mathrm{Si}$ is due to the softened optical modes near the zone center caused by the boron doping [16]. The electron phonon coupling constant, $\lambda$, was estimated to be $0.2-0.3$, where the superconductivity above $1 \mathrm{~K}$ requires the hole doping over $5 \%$, which corresponds to the hole density of $2.8 \times 10^{21} \mathrm{~cm}^{-3}[16,17]$. Thus, the electric-fieldinduced hole density of the $\operatorname{Si}(110)$ surface is not enough to provide the hole density to excite superconductivity. 


\section{CONCLUSION}

We investigated the electronic structures and the induced hole carriers of the hydrogenated $\mathrm{Si}(110)$ surface in an electric field by means of first-principles calculations. The electric field introduces the metallic character at the surface where the Fermi surface with the hole pocket appears at the center of the $\Gamma$ point. The hole carriers are induced up to $1.1 \times 10^{21} \mathrm{~cm}^{-3}$ at the $\mathrm{Si}(2)$ layer, which are less than the critical hole density of the boron-doped
Si superconductivity, and the $\mathrm{Si}(110)$ surface may be difficult to excite superconductivity by the electric field.

\section{Acknowledgments}

Work was supported by Grant-in Aid for Scientific Research (Nos. 23540405 and 20540334) from the Japan Society for the Promotion of Science. Computations were partially performed at ISSP, University of Tokyo.
[1] E. A. Ekimov, V. A. Sidorov, E. D. Bauer, N. N. Mel'nik, N. J. Curo, J. D. Thompson, and S. M. Stishov, Nature 428, 542 (2004).

[2] Y. Takano, T. Takenouchi, S. Ishii, S. Ueda, T. Okutsu, I. Sakaguchi, H. Umezawa, H. Kawarada, and M. Tachiki, Diamond Relat. Mater. 16, 911 (2007).

[3] E. Bustarret, C. Marcenat, P. Achatz, J. Kačmarčik, F. Lévy, A. Huxley, L. Ortéga, E. Bourgeois, X. Blasé, D. Débarre, and J. Boulmer, Nature (London) 444, 465 (2006).

[4] Z.-A. Ren, J. Kato, T. Muranaka, J. Akimitsu, M. Kriener, and Y. Maeno J. Phys. Soc. Jpn. 76, 103710 (2007).

[5] X. Blase, E. Bustarret, C. Chapelier, T. Klein, and C. Marcenat, Nat. Mater. 8, 375 (2009).

[6] E. Bourgeois, E. Bustarret, P. Achatz, F. Omnés, and X. Blasé, Phys. Rev. B 74, 094509 (2006).

[7] J. P. Goss and P. R Briddon, Phys. Rev. B 73, 085204 (2006).

[8] K. Ueno, S. Nakamura, H. Shimotani, A. Ohtomo, N. Kimura, T. Nojima, H. Aoki, Y. Iwasa, and M. Kawasaki,
Nat. Mater. 7, 855 (2008)

[9] K. Nakamura, S. H. Rhim, A. Sugiyama, K. Sano, T. Akiyama, T. Ito, M. Weinert, and A. J. Freeman, Phys. Rev. B 87, 214506 (2013).

[10] E. Wimmer, H. Krakauer, M. Weinert, And A. J. Freeman, Phys. Rev. B 24, 864 (1981).

[11] M. Weinert, E. Wimmer, and A. J. Freeman, Phys. Rev. B 26, 4571 (1982).

[12] K. Nakamura, T. Ito, A. J. Freeman, L. Zhong, and J. Fernandez-de-Castro, Phys. Rev. B 67, 014420 (2003).

[13] U. von Barth and L. Hedin, J. Phys. C 5, 1629 (1972).

[14] K. Nakamura, R. Shimabukuro, Y. Fujiwara, T. Akiyama, T. Ito, and A. J. Freeman, Phys. Rev. Lett. 102, 187201 (2009).

[15] M. Weinert, G. Schneider, R. Podlouky, and J. Redinger, J. Phys.: Condens. Matter 21, 084201 (2009).

[16] E. Bourgeois and X. Blase, Appl. Phys. Lett. 90, 142511 (2007).

[17] L. Borei, J. Kortus, and O. K. Anderson, Phys. Rev. Lett. 93, 237002 (2004). 